\title{
Variations in prostate biopsy practice: A quantitative questionnaire- based study
}

\author{
Matthew O. Lipinski; ${ }^{*}$ D. Robert Siemens, MD, FRCSC; ${ }^{\dagger}$ Patti A. Groome, PhD*
}

*Division of Cancer Care and Epidemiology, Cancer Research Institute; and the Department of Community Health and Epidemiology, Queen's University, Kingston, ON; 'Department of Oncology; and the Department of Urology, Queen's University, Kingston, ON

Cite as: Can Urol Assoc J 2013;7(11-12):e732-9. http://dx.doi.org/10.5489/cuaj.372

Published online November 8, 2013.

\section{Abstract}

Background: The ambivalent results of recent large randomized prostate cancer studies have added a significant layer of uncertainty for clinicians and patients contemplating investigation of early disease. This uncertainty and lack of prescriptive recommendations from professional organizations has led to significant variation in practice in North America. The purpose of this study was to determine the extent of variation in biopsy recommendations by urologists and to discern factors predictive for these recommendations. Methods: An anonymous, cross-sectional, self-report questionnaire was sent to all active members of the Canadian Urological Association practicing in Ontario. The survey consisted of demographic data and 10 closed-ended questions designed to capture biopsy preferences in ambiguous clinical situations. Respondent preferences for recommending a prostate biopsy were compared to a guideline-informed study standard. Descriptive and correlative statistics were used to analyze the responses.

Results: The response rate to the survey was $74 \%$. The responses showed considerable variability in recommendations for or against biopsy. While most of the urologists concurred with the research team's study standard recommendations, only 4 scenarios had over $80 \%$ concurrence and 1 scenario, which centered on the utility of free PSA, had only had $42 \%$ concurrence. None of the respondent's descriptors were associated with trends to recommend biopsy other than the number of biopsies performed per year $(p=0.04)$.

Interpretation: This self-report survey investigating prostate biopsy thresholds identifies considerable variation in practicing urologists in Ontario. The drivers of biopsy recommendations in these relatively ambiguous clinical situations appeared to be age, suspicious rectal examinations, and total PSA.

\section{Introduction}

Prostate cancer is the most commonly diagnosed cancer in western countries and represents a significant healthcare burden in Canada; 1 in 6 men will be diagnosed with the disease in their lifetime. ${ }^{1,2}$ Despite its prevalence and associated morbidity and mortality, early detection remains controversial, most recently due to conflicting messages from 2 pivotal randomized trials $s^{3,4}$ and because of the potential for over-diagnosis and overtreatment of men with indolent prostate cancer. ${ }^{3}$ Many national organizations and advocacy groups, including the Canadian Urological Association (CUA), have published guidelines regarding prostate-specific antigen (PSA) testing. ${ }^{5,6}$ They explain that the presence of prostate cancer is not indicated by a single PSA cutoff point but, instead, that a higher PSA level is associated with a higher risk of disease. ${ }^{7}$ Therefore, these guidelines stress the importance of shared decision-making between the patient and his doctor. ${ }^{6,7}$ Because of the ambiguity of this recommendation, there is significant variation in screening practice across multiple factors that are not clinically relevant, such as geographic location, remuneration mechanism, and personal opinion. ${ }^{7-11}$

Ultimately, any realized benefit of PSA screening, as well as potential harm, will rest on the decision to perform a transrectal ultrasound and biopsy (TRUS-BX). However, uncertainty about PSA result interpretation leads to uncertainty about when to biopsy. ${ }^{6,7}$ Although it would appear that, in Canada, the main driver of TRUS-BX is patient age relative to total PSA values, ${ }^{11}$ other factors add to the clinical complexity and these need to be considered in the decision to biopsy. These factors include: PSA levels relative to prostate volume, PSA kinetics, family history, ethnicity, and comorbidities. ${ }^{6}$ The situation has recently been amplified by findings such as those from the prostate cancer prevention trial (PCPT), ${ }^{12}$ indicating that historical cutoff levels for prostate cancer screening using a PSA level of $4 \mathrm{ng} / \mathrm{mL}$ may no longer be appropriate, particularly among younger men. Lastly, the decision to biopsy needs to be weighed against the complication rates of TRUS-BX, ${ }^{13-19}$ particularly with more recent understanding of the frequency of serious infectious complications requiring hospitalization. ${ }^{18}$ 
Given the controversy around optimal screening and diagnosis of early prostate cancer, as well as the ambivalent recommendations from national and international bodies, we expected that, in this post-screening trial era, there would be important differences among urologists about who is offered biopsy which could lead to significant differences in quality of prostate cancer care. Our aim was to document current biopsy recommendations based on different clinical scenarios.

\section{Methods}

We surveyed all active Ontario members of the CUA for their views on when to biopsy the prostate. Our mailed survey was designed to capture biopsy preferences in ambiguous clinical situations using a 5-point Likert scale anchored at "recommend against biopsy" and "recommend for biopsy." The questions encompassed 10 scenarios that varied regarding factors that could affect the urologist's decision (Table 1). We consulted multiple practice guidelines to relate our scenarios to standard practice regarding investigation recommendations and we used those guidelines along with clinical experience (RS) to state explicit choices for or against biopsy for each scenario (Table 1). 5,6,20-24 We also collected 7 descriptors of the urology participants that might have been associated with their biopsy practice: age, sex, number of years since graduation, whether they do prostate biopsies and/or prostatectomies, hospital type, and location. The full survey is provided in Appendix 1.

We used a modified Dillman method to maximize survey response. Specifically, we sent a second mailing 1 month after the initial mailing to all who had not yet completed the questionnaire. ${ }^{25}$ We stopped accepting responses 1 month after the second mailing. The initial questionnaire package included a pre-addressed, pre-stamped return envelope and a $\$ 5$ gift certificate. Data were anonymized using a unique questionnaire identification number and were recorded in a Microsoft Office Access 2007 database.

We presented the Likert-scale distributions for each scenario and indicated which response agreed with the study

\section{Table 1. Study team consensus recommendations regarding biopsy for clinical scenarios}

Case scenarios Clinical drivers

1. Healthy 47-year-old male with positive family - Life expectancy $>10$ years

history of prostate cancer (father was diagnosed at - Positive family history

62). DRE negative and PSA $1.7 \mathrm{ng} / \mathrm{mL}$

2. Healthy 55-year-old male with no family history of prostate cancer and DRE negative. Total PSA 2.7 $\mathrm{ng} / \mathrm{mL}$ last year and $2.8 \mathrm{ng} / \mathrm{mL}$ this year

3. Healthy 72-year-old male with negative DRE. Total PSA stable at $4.8 \mathrm{ng} / \mathrm{mL}$

4. Healthy 66-year-old male with negative DRE. PSA values from the last 3 annual tests $3.9,4.2$, and 4.1 $\mathrm{ng} / \mathrm{mL}$, but free/total ratio $10 \%$

5. Healthy 74-year-old male with stable total PSA 3.4$3.6 \mathrm{ng} / \mathrm{mL}$, but suspicious DRE on right lobe of the prostate

6. 68-year-old male who had a coronary artery bypass graft 2 years ago and is a cigarette smoker. Total PSA was $5.6-7.2 \mathrm{ng} / \mathrm{mL}$ and negative DRE

7. 59-year-old male with total PSA values in previous annual tests of $2.4,2.6,3.8$, and $3.9 \mathrm{ng} / \mathrm{mL}$. DRE negative.

8. 51-year-old African-Canadian male with total PSA value between 3.5 and $3.8 \mathrm{ng} / \mathrm{mL}$. DRE negative

9. Healthy 57-year-old male with a prostate volume of $30 \mathrm{cc}$. Total PSA value $2 \mathrm{ng} / \mathrm{mL}$ and DRE negative

10.61-year-old male with a BMI 30 (obese). Total PSA $3.1 \mathrm{ng} / \mathrm{mL}$ and DRE negative
- Low PSA

- Life expectancy $>10$ years

- Low PSA for age

- Low PSA velocity

- Age over 70

- Life expectancy $>10$ years

- Low PSA velocity

- PSA equivocal for age

- Life expectancy >10 years

- Low PSA velocity

- PSA equivocal for age

- Low free/total PSA

- Age over 70

- Life expectancy potentially >10 years

- PSA low for age

- Suspicious DRE

- Life expectancy potentially $<10$ years

- PSA slightly elevated for age

- PSA slightly elevated for age

- PSA velocity equivocal

- Potential increased risk due to ethnicity

- Life expectancy >10 years

- PSA slightly elevated for age

- Life expectancy $>10$ years

- PSA low

- Low PSA density

- Life expectancy potentially $<10$ years

- PSA equivocal for age

- Potential increased risk due to BMI

\section{Investigative outcome}

Favour recommending against biopsy

Favour recommending against biopsy

Favour recommending against biopsy

Favour recommending for biopsy

Favour recommending for biopsy

Favour recommending for biopsy

Favour recommending for biopsy

Favour recommending for biopsy

Favour recommending against biopsy

Favour recommending against biopsy

DRE: digital rectal examination; PSA: prostate-specific antigen; BMI: body mass index. 
Lipinski et al.

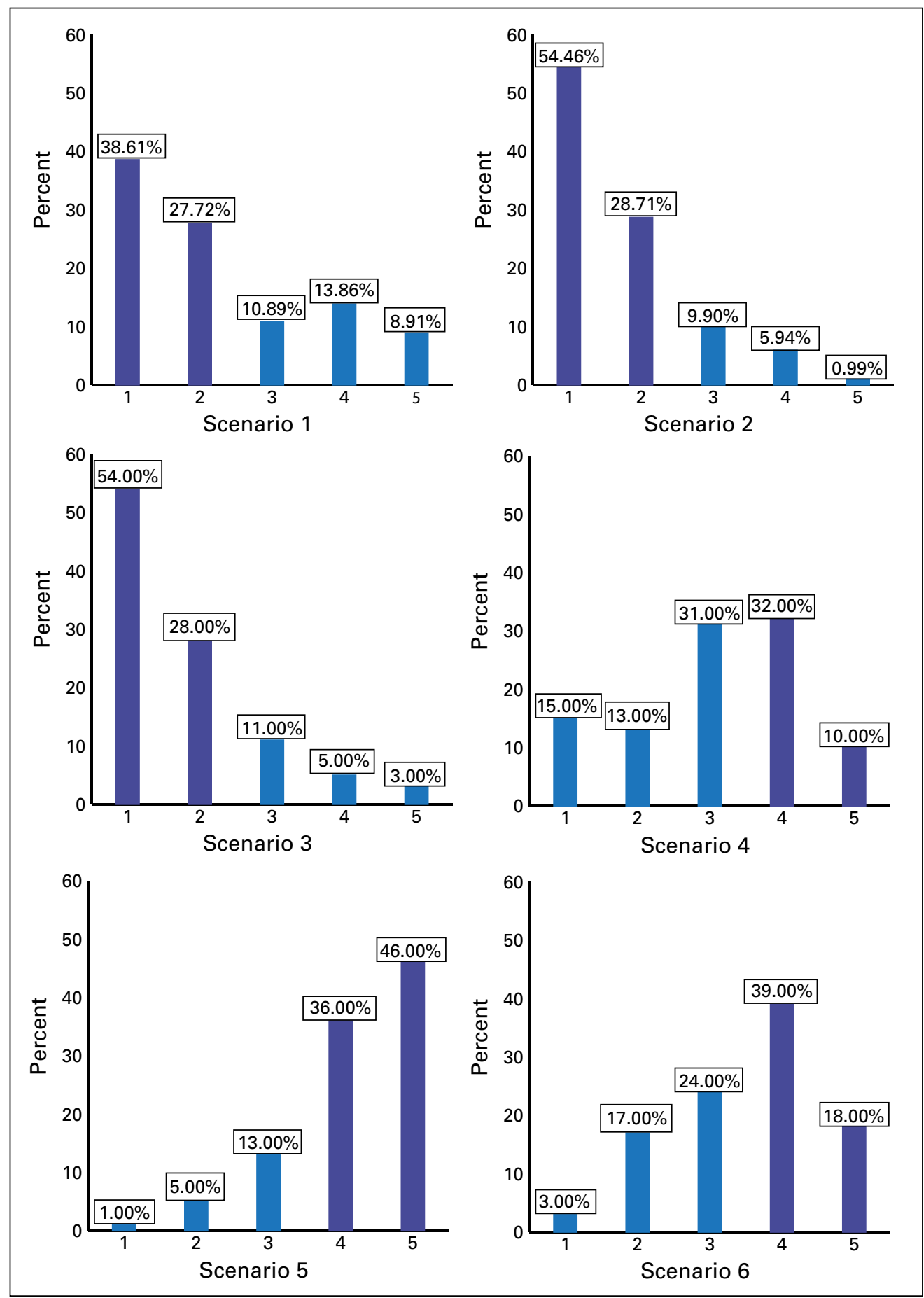

Fig. 1. Frequency distribution of Likert scale responses to 10 clinical scenarios. Those frequencies agreeing with our clinical assessment are shown in dark blue. 1 or 2=recommend against biopsy, 4 or 5 =recommend for biopsy.

team's assignment for or against a prostate biopsy. We also calculated the overall average Likert score across the 10 scenarios for each respondent as a measure of the respondent's propensity to biopsy. We correlated these scores with respondent age, years since graduation of medical school, reported annual number of biopsies and prostatectomies performed, and practice setting by calculating Pearson's correlation coefficient for continuous factors and conducting an analysis of variance for categorical factors. Analyses were conducted using SPSS PASW Statistics 18 and SAS version 9.2 (SAS Institute, Cary, NC). 


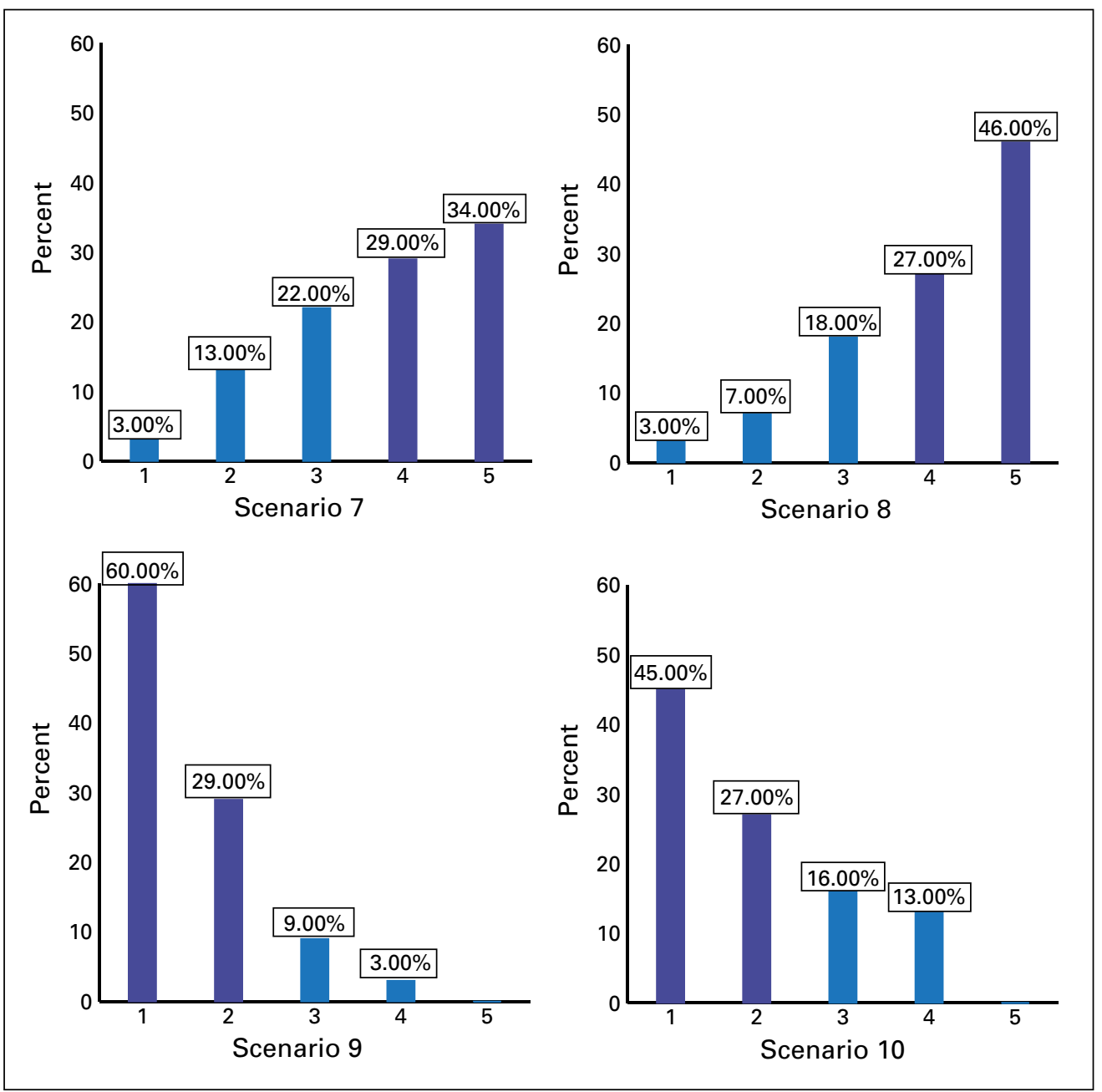

\section{Results}

Of the original 148 questionnaires sent, 108 eligible responses were received and 2 had moved out of the province for an overall response rate of $74.0 \%$. Of those responses, 101 answered all of the 10 clinical scenarios (Table 2). In total, $57 \%$ of respondents were in community hospitals, $24 \%$ performed more than 140 biopsies per year and more than 30 prostatectomies. Over $52 \%$ reported performing no biopsies, likely due to biopsy referrals made to radiology or other urology colleagues.

We illustrated the distribution of Likert responses for each of the 10 clinical scenarios (Fig. 1). We highlighted the responses that agree with our guideline-based assignment about whether a biopsy was warranted by taking a response of 1 or 2 as representing recommendation against biopsy and a response of 4 or 5 as representing recommendation for biopsy. Most concurred with this assignment, but the degree of concurrence varied from a low of $42 \%$ for scenario 4 to a high of $89 \%$ for scenario 9 . Over $80 \%$ concurred with our assignment against biopsy for scenarios 2, 3, and 9 and with our assignment for biopsy in scenario 5. Agreement with our assignment exceeded $70 \%$ in scenarios 8 and 10 .

The average summed biopsy score across all 10 scenarios was 27.9 (standard deviation 5.6, range: 17-40) out of a possible total score of 50 . None of the urologist descriptors were associated with the average biopsy scores except for the average annual number of biopsies performed, which showed an increase of 3.4 in the propensity to biopsy score across the 3 categories $(p=0.04)$ (Table 3 ).

\section{Discussion}

Active members of the CUA from Ontario were sent a questionnaire about their opinion to recommend for or against biopsy in 10 hypothetical cases. We achieved a response rate of $74 \%$, which is high for such surveys. We take this as an indication of the level of interest in this topic. With this response, we interpret our results as providing a population-based view of the variations in opinion about when to 


\begin{tabular}{lc}
\hline Table 2. Respondent characteristics & \\
\hline Sex, $\%(n=100)$ & \\
Male & 99.0 \\
Female & 1.0 \\
Age $(n=98)$ & $51.2(9.7)$ \\
$\quad$ Mean (SD) & \\
Years since graduation ( $\mathrm{n}=98)$ & $24.8(10.2)$ \\
$\quad$ Mean (SD) & \\
Hospital type, \% ( $=100)$ & 57.0 \\
Community & 40.0 \\
Teaching & 3.0 \\
Other & \\
No. biopsies per year, \% ( $=99)$ & 52.5 \\
0 & 23.2 \\
1-140 & 24.2 \\
141-900 & \\
No. prostatectomies per year, \% ( $=99)$ & 26.3 \\
0 & 26.3 \\
1-20 & 23.2 \\
21-30 & 24.2 \\
31-90 & \\
\hline SD: standard deviation. &
\end{tabular}

conduct a prostate biopsy in ambiguous clinical situations. Perhaps not surprisingly, urologists who conduct a lot of biopsies had a higher propensity to biopsy such cases.

While most respondents agreed with our guidelineinformed study assignments to recommend for or against biopsy, there was a sizeable number who did not. This lack of consensus could be largely due to the variations in guidance that is being provided by the various standard setting bodies $5,6,20,21,24$ and the challenge of choosing and/ or using such guidance in everyday clinical practice. Our stated biopsy recommendations were explicit; we asked respondents if they were in favour of or against biopsy in an attempt to come to an actual decision, regardless of the ambiguity of the case (Table 1), representing informed, albeit arbitrary, decisions. It is interesting to note that in this study, the clinical drivers in those scenarios with the greatest concordance were age, suspicious digital rectal examination and a low PSA value (particularly a PSA between 2.0 and 3.0). This pattern is observed despite the well-described incidence of prostate cancer (nearly $24 \%$ ) in the PCPT trial for PSA values in this range. ${ }^{12}$ Ethnicity (scenario 8 ) influenced respondent's biopsy recommendations toward favouring biopsy; obesity, on the other hand, a putative risk factor for prostate cancer, did not strongly influence the recommendations in the presence of the competing concern of life expectancy/comorbidities (scenario 10) - most respondents recommended against biopsy. The greatest discordance with the study team's designated standard recommendation was for scenario 4 at $42 \%$ exploring the role of free PSA in the decision to biopsy.

Urologists' clinical decisions may be influenced by previous personal or anecdotal experience in addition to guide-
Table 3. Respondent characteristic associations with summed biopsy score

\begin{tabular}{lcc}
\hline Characteristic & $\begin{array}{c}\text { Correlations with biopsy } \\
\text { score* }\end{array}$ & $\boldsymbol{p}$ value \\
\hline Age & -0.07 & 0.47 \\
Years since graduation & -0.10 & 0.34 \\
\hline Characteristic & Mean biopsy score (SD) & $\boldsymbol{p}$ value \\
\hline Hospital type & & 0.36 \\
$\quad$ Community & $27.6(5.6)$ & \\
Teaching & $28.7(5.5)$ & \\
No. biopsies per year & & 0.04 \\
0 & $26.8(5.0)$ & \\
1-140 & $27.7(6.4)$ & \\
141-900 & $30.2(5.5)$ & \\
No. prostatectomies per year & & \\
0 & $27.3(5.7)$ & \\
1-20 & $27.3(5.6)$ & \\
21-30 & $27.0(4.9)$ & \\
$31-90$ & $29.6(6.1)$ & \\
\hline SD: standard deviation; *Pearson r. & & \\
\hline
\end{tabular}

lines, especially in the presence of inconclusive advice. In the United Kingdom, Burden and colleagues surveyed 733 conference attendee urologists on their usage of guidelines about the usage of PSA tests and subsequent biopsy investigations. In that study, of the $47 \%$ who responded, there was a wide variation of their use of guidelines and $42 \%$ were not aware of the current PSA cut-off recommendations. ${ }^{26}$ Similarly, Lawrentschuk and colleagues conducted a survey of CUA members to document what PSA levels lead to a recommendation for ultrasound guided biopsy and to explore if there were other factors that influenced further investigations. Of the 360 urologists surveyed, the $35 \%$ who responded indicated a considerable amount of variation with each of their scenarios. Their use of a factorial design identified age and PSA as influential to practice. ${ }^{11}$ Our study adds to this work in that it explored urologists' viewpoints about more ambiguous, multifactorial cases. Also, our results reflect urologists' preferences after the release of the Prostate, Lung, Colorectal, and Ovarian (PLCO) and European Randomized Study of Screening for Prostate Cancer (ERSPC) screening study findings.

Our results are restricted to Ontario urologists who were active members of the CUA because of limited time, funding, and mailing list availability. Active CUA members, who make up about $75 \%$ of all active Ontario urologists, may be more likely to follow guidelines thereby increasing our findings on guideline concordance. Restriction to Ontario may have reduced the amount of practice variation we observed compared to what the results of a Canada-wide survey might have revealed. Our results do not reflect the views of the $26 \%$ who did not respond. We were unable to compare characteristics of non-respondents to provide information about the representativeness of our study population. We did not use a factorial design, which would have involved 
many more than 10 scenarios, to isolate the various patient factors that might have influenced biopsy assignments and we minimized questions about respondent's training. These were deliberate decisions made to maximize our response rate by keeping the questionnaire short.

\section{Conclusion}

We collected information about prostate biopsy clinical decision-making from a large sample of urologists from across Ontario. We documented that this decision-making varies and we speculated that this variation is due to a lack of clear practice guidance in this area. Further studies should try and determine the specific aspects of a clinical case that may underlie these variations and specifically probe the rationale for decision-making. This information could inform professional organizations and advocacy groups developing guidelines or recommendations regarding the relative appropriateness of prostate biopsy.

Acknowledgement: This work was supported by the Hope for Life campaign.

Competing interests: Dr. Siemens is Editor-in-chief at CUAJ and he along with Dr. Lipinski and Dr. Groome declare no other competing financial or personal interests.

This paper has been peer-reviewed.

\section{References}

1. Canadian Cancer Society's Steering Committee. Canadian Cancer Statistics 2010. Toronto: Canadian Cancer Society; 2010.

2. Prostate Cancer Research Foundation of Canada. Prostate Cancer Canada; 2010. http://www.prostatecancer.ca/Prostate-Cancer/About-Prostate-Cancer\#.Um7NkflQHIY. Accessed October 28, 2013.

3. Schroder FH, Hugosson J, Roobol MJ, et al. Screening and prostate-cancer mortality in a randomized European study. N Engl J Med 2009;360:1320-8. http://dx.doi.org/10.1056/NEJMoa0810084

4. Andriole GL, Crawford ED, Grubb RL 3rd, et al. Mortality results from a randomized prostate-cancer screening trial. N Engl J Med 2009;360:1310-9. http://dx.doi.org/10.1056/NEJMoa0810696

5. Carroll P, Albertsen P, Greene K, et al. Prostate Specific Antigen Best Practice Statement: 2009 Update. American Urological Association Education and Research Inc 2009. http://www.auanet.org/common/ pdf/education/clinical-guidance/Prostate-Specific-Antigen.pdf. Accessed October 28, 2013.

6. Canadian Urological Association. Prostate cancer screening: Canadian guidelines 2011. Can Urol Assoc J 2011;5:235-40. http://dx.doi.org/10.5489/cuaj.11134

7. Bunting PS, Goel V, Williams II, et al. Prostate-specific antigen testing in Ontario: reasons for testing patients without diagnosed prostate cancer. CMAJ 1999;160:70-5.

8. Austin 0J, Valente S, Hasse LA, et al. Determinants of Prostate-Specific Antigen Test Use in Prostate Cancer Screening by Primary Care Physicians. Arch Fam Med 1997;6:453-8. http://dx.doi.org/10.1001/ archfami.6.5.453
9. Drummond FJ, Carsin AE, Sharp L, et al. Factors prompting PSA-testing of asymptomatic men in a country with no guidelines: a national survey of general practitioners. BMC Fam Pract 2009;10:3. http://dx.doi. org/10.1186/1471-2296-10-3

10. Linder $S$, Hawley $S$, Cooper $C$, et al. Primary care physicians' reported use of pre-screening discussions for prostate cancer screening: a cross-sectional survey. BMC Fam Pract 2009;10:19. http://dx.doi. org/10.1186/1471-2296-10-19

11. Lawrentschuk N, Dalieet N, Ma C, et al. Prostate-specific antigen test result interpretation when combined with risk factors for recommendation of biopsy: a survey of urologist's practice patterns. Int Urol Nephrol 2011;43:31-7. http://dx.doi.org/10.1007/s11255-010-9772-1

12. Thompson IM, Ankerst DP, Chi C, et al. Assessing prostate cancer risk: results from the Prostate Cancer Prevention Trial. J Natl Cancer Inst 2006;98:529-34. http://dx.doi.org/10.1093/inci/diil31

13. de Jesus $C M$, Correa LA, Padovani CR. Complications and risk factors in transrectal ultrasound-guided prostate biopsies. Sao Paulo Med J 2006;124:198-202. http://dx.doi.org/10.1590/S151631802006000400005

14. Deliveliotis C, John V, Louras $G$, et al. Multiple transrectal ultrasound guided prostatic biopsies: morbidity and tolerance. Int Urol Nephrol 1999;31:681-6. http://dx.doi.org/10.1023/A:1007168823851

15. Raaiimakers R, Kirkels WJ, Roobol MJ, et al. Complication rates and risk factors of 5802 transrectal ultrasound-guided sextant biopsies of the prostate within a population-based screening program. Urology 2002;60:826-30. http://dx.doi.org/10.1016/S0090-4295(02)01958-1

16. Tuncel A, Kirilmaz U, Nalcacioglu $V$, et al. The impact of transrectal prostate needle biopsy on sexuality in men and their female partners. Urology 2008;71:1128-31. http://dx.doi.org/10.1016/i. urology.2008.01.055

17. Chiang IN, Chang SJ, Pu YS, et al. Major complications and associated risk factors of transrectal ultrasound guided prostate needle biopsy: a retrospective study of 1875 cases in taiwan. J Formosan Med Assoc 2007;106:929-34. http://dx.doi.org/10.1016/S0929-6646(08)60063-7

18. Nam RK, Saskin R, Lee $Y$, et al. Increasing hospital admission rates for urological complications after transrectal ultrasound guided prostate biopsy. J Urol 2010;183:963-8. http://dx.doi.org/10.1016/i. juro.2009.11.043

19. Roberts RO, Bergstralh EJ, Besse JA, et al. Trends and risk factors for prostate biopsy complications in the pre-PSA and PSA eras, 1980 to 1997. Urology 2002:59:79-84. http://dx.doi.org/10.1016/ S0090-4295(01)01465-0

20. Stricker P, Phelps K. Prostate Cancer for the General Practitioner: PSA booklet edition I; 2004.

21. Bauman G. Prostate Cancer: GU Guideline 2008:20.

22. Calle EE, Rodriguez C, Walker-Thurmond $\mathrm{K}$, et al. Overweight, obesity, and mortality from cancer in a prospectively studied cohort of U.S. adults. N Eng/J Med 2003;348:1625-38. http://dx.doi.org/10.1056/ NEJMoo021423

23. Finne $\mathrm{P}$, Auvinen $\mathrm{A}$, Aro J, et al. Estimation of prostate cancer risk on the basis of total and free prostate-specific antigen, prostate volume and digital rectal examination. Eur Urol 2002;41:619-26.; discussion 626-7.

24. Prostate Cancer Early Detection Version 2.2012. NCCN Clinical Practice Guidelines in Oncology National Comprehensive Cancer Network 2011.

25. Aday LA. Chapter 13: Monitoring and Carrying Out the Survey. Designing and Conducting Health Surveys. Second Edition ed. San Francisco, CA: Jossey-Bass; 1996:281.

26. Burden $H P$, Davis $C R$, Tate $S$, et al. The trends in prostate specific antigen usage amongst United Kingdom urologists-a questionnaire based study. BMC Urol 2008;8:17. http://dx.doi.org/10.1186/1471-24908-17

Correspondence: Dr. Patti Groome, Division of Cancer Care and Epidemiology, Cancer Research Institute, 10 Stuart Street, 2nd Level, Kingston, Ontario, Canada K7L 3N6; fax: 613-533-6794; patti.groome@krcc.on.ca 


\begin{abstract}
Appendix 1.
Dear "Title» «Fname» "Lname»,

The Department of Urology at Queen's University and the Division of Cancer Care and Epidemiology in the Queen's Cancer Research Institute is conducting a paper patient survey designed to describe the prostate biopsy practice patterns of urologists practicing in Ontario. Our goal is to describe the factors that affect urologists' decision to recommend a prostate biopsy in the presence of ambiguous screening results. The information from this survey will also be correlated with county-level prostate biopsy rates. Your participation is extremely important to the success of this project and therefore, greatly appreciated.
\end{abstract}

This questionnaire should take no longer than $\mathbf{1 0}$ minutes to complete. It is also important for us to know if you do not perform prostate biopsies. So if that is the case, please complete only the demographic questions on the final page and return the (otherwise) blank questionnaire.

Your participation in this survey is voluntary and your answers will remain anonymous. No identifying information will be collected that could lead to linking back to your responses. Our published findings will be aggregated to protect the confidentiality of those responses.

Please accept the enclosed $\$ 5$ gift card as a thank you for taking time to read and (hopefully) complete this study. Your input is important for helping to further our understanding of practice decisions and patterns in prostate cancer across Ontario.

This survey has been reviewed and approved by the Ethics Review Board at Queen's University. If you have any concerns about your rights as a research subject please contact Dr. Albert Clark, Chair of the Queen's University Health Sciences and Affiliated Teaching Hospitals Research Ethics Board at (613) 533-6081.

Once you have completed the survey, please mail it back in the enclosed self-addressed stamped envelope.

We would like to thank you again for your time and help with this study.

Yours Sincerely,

Matt, Rob and Patti

Matthew Lipinski matthew.lipinski@queensu.ca

Dr. Rob Siemens siemensr@kgh.kari.net

Tel: (613) 549-6666 ext. 2411

Dr. Patti Groome patti.groome@krcc.on.ca

Tel: (613) 533-6000 ext. 78512

\title{
For the following 10 cases please indicate your recommendation for biopsy from 1 (Recommend against biopsy)
} to 5 (Recommend for biopsy).

1. Healthy 47-year-old male with positive family history of prostate cancer (father was diagnosed at 62 ).

DRE was negative and PSA is $1.7 \mathrm{ng} / \mathrm{mL}$.

\section{Recommend against}

biopsy

1

\section{Recommend for biopsy}

5

Recommend for biopsy

5

\section{Recommend for biopsy}

5

4. Healthy 66-year-old male with negative DRE. PSA values from the last three annual tests have been $3.9,4.2,4.1 \mathrm{ng} / \mathrm{mL}$ but Free/Total ratio is $10 \%$.

Recommend against biopsy

1

2

4

5. Healthy 74-year-old male with stable Total PSA of $3.4-3.6 \mathrm{ng} / \mathrm{mL}$ but suspicious DRE on right lobe of the prostate.

Recommend against

biopsy

1
2

\section{Recommend for biopsy}


6. 68-year-old male who had a coronary artery bypass graft 2 years ago and is a cigarette smoker. Total PSA has been 5.6-7.2 $\mathrm{ng} / \mathrm{mL}$ and negative DRE.

Recommend against biopsy

1 2

3

4

\section{Recommend for biopsy}

5

7. 59-year-old male with total PSA values in previous annual tests of $2.4,2.6,3.8$, and $3.9 \mathrm{ng} / \mathrm{mL}$. DRE was negative. Recommend against biopsy

1 2 3 4

Recommend for biopsy

5

Recommend for biopsy

5

Recommend for biopsy 5

10.61-year-old male with a Body mass Index (BMI) of 30 (Obese Category). The total PSA was $3.1 \mathrm{ng} / \mathrm{mL}$ and DRE was negative. Recommend against biopsy 1 2 3 4 biopsy 5

All respondents (including those that do not perform prostate biopsies): Please complete the following $\underline{7}$ demographic questions:

1. Age:

2. Sex:

3. Years Since Graduation from Medical School:

4. Do you perform prostate biopsies and/or prostatectomies? (If only one, please indicate)

Yes / No

5. If you answered yes to question 4, approximately how many do you perform in a year?

Prostate biopsies:

Prostatectomies:

6. What type of practice do you work in (circle one)?

Community Hospital Teaching Hospital Clinic Other

If other, please specify:

7. In what county do you practice?

(If you don't know the name of the county, please indicate the city you practice in)

Thank you very much for taking the time to complete this survey

Please return within the enclosed self-addressed envelope to:

QUEEN'S CANCER RESEARCH INSTITUTE

DIVISION OF CANCER CARE

AND EPIDEMIOLOGY

10 Stuart Street, Level 2

Kingston, Ontario, Canada K7L 3N6 